\title{
Linearly Polarized, Single-Mode Spontaneous Emission in a Photonic Nanowire
}

Munsch, Mathieu; Claudon, Julien; Bleuse, Joël; Malik, Nitin S.; Dupuy, Emmanuel; Gerard, Jean-Michel; Chen, Yuntian; Gregersen, Niels; Mørk, Jesper

Published in:

Physical Review Letters

Link to article, DOI:

10.1103/PhysRevLett.108.077405

Publication date:

2012

Document Version

Publisher's PDF, also known as Version of record

Link back to DTU Orbit

Citation (APA):

Munsch, M., Claudon, J., Bleuse, J., Malik, N. S., Dupuy, E., Gerard, J-M., Chen, Y., Gregersen, N., \& Mørk, J. (2012). Linearly Polarized, Single-Mode Spontaneous Emission in a Photonic Nanowire. Physical Review Letters, 108(7), 077405. https://doi.org/10.1103/PhysRevLett.108.077405

\section{General rights}

Copyright and moral rights for the publications made accessible in the public portal are retained by the authors and/or other copyright owners and it is a condition of accessing publications that users recognise and abide by the legal requirements associated with these rights.

- Users may download and print one copy of any publication from the public portal for the purpose of private study or research.

- You may not further distribute the material or use it for any profit-making activity or commercial gain

- You may freely distribute the URL identifying the publication in the public portal 


\title{
Linearly Polarized, Single-Mode Spontaneous Emission in a Photonic Nanowire
}

\author{
Mathieu Munsch, Julien Claudon, ${ }^{*}$ Joël Bleuse, Nitin S. Malik, Emmanuel Dupuy, and Jean-Michel Gérard \\ CEA-CNRS-UJF group "Nanophysique et Semiconducteurs", CEA, INAC, SP2M, F-38054 Grenoble, France \\ Yuntian Chen, Niels Gregersen, and Jesper Mørk \\ DTU Fotonik, Department of Photonics Engineering, Technical University of Denmark, Building 343, \\ DK-2800 Kongens Lyngby, Denmark \\ (Received 20 September 2011; published 17 February 2012)
}

\begin{abstract}
We introduce dielectric elliptical photonic nanowires to funnel efficiently the spontaneous emission of an embedded emitter into a single optical mode. Inside a wire with a moderate lateral aspect ratio, the electromagnetic environment is largely dominated by a single guided mode, with a linear polarization oriented along the ellipse major axis. The resulting monomode spontaneous emission is maintained over a broad wavelength range, a key asset of this $1 \mathrm{D}$ photonic structure. Our theoretical analysis is completed by an experimental study of GaAs elliptical photonic wires with embedded InAs quantum dots. In particular, the fraction of collected photons with the desired linear polarization can exceed $95 \%$.
\end{abstract}

DOI: 10.1103/PhysRevLett.108.077405

PACS numbers: 78.67.Pt, 78.55.Cr, 78.67.Hc

Since the pioneering work of Purcell (p. 681 of Ref. [1]), it has been recognized that the spontaneous emission (SE) of an emitter can be manipulated to a large extent through a proper engineering of its electromagnetic environment. Ultimately, all the SE can be funneled into a single optical mode, with a well defined spatial structure and polarization. Realizing this situation in the solid-state holds great promise for the development of a new generation of advanced optoelectronic devices, like ultralow threshold microlasers [2,3] and efficient single-photon sources with polarization control [4-6]. More generally, the coupling of a single quantum emitter to a single electromagnetic mode, referred as a 1D atom [7], constitutes a model system for quantum optics experiments and lies at the root of advanced quantum information processing networks [8].

Inspired by the remarkable achievements in atomic physics, preferential emission into a single localized mode has been first achieved taking advantage of the Purcell effect that arises in semiconductor optical microcavities $[4,6,9]$. Recently, the need to overcome the limited operation bandwidth inherent to a resonant approach has triggered intense research on SE control in waveguide structures. Various platforms have been investigated experimentally, including semiconductor ring or fiberlike photonic nanowires [10,11], waveguides defined in a photonic crystal membrane $[12,13]$, or plasmonic waveguides [14]. Among these candidates, cylindrical fiberlike photonic nanowires are particularly attractive, as shown by the recent development of a very bright single-photon source $[11,15]$.

However, for simple symmetry reasons, a photonic wire with a circular section always supports two degenerate guided modes with orthogonal linear polarization. In these conditions, single-mode emission can only be achieved using a highly polarized emitter, which is not the case for usual semiconductor emitters [e.g., neutral, charged excitons trapped in a quantum dot (QD) or excitons confined in a quantum well $(\mathrm{QW})]$. In this Letter, we lift this limitation by implementing photonic nanowires featuring an anisotropic cross section. Calculations show that even a moderate $(\sim 2)$ lateral aspect ratio leads to a very large optical anisotropy. Inside such a wire, the electromagnetic environment is largely dominated by the contribution of a single guided mode whose linear polarization is oriented along the ellipse major axis. Polarization-resolved measurements conducted on elliptical GaAs photonic nanowires embedding spectrally isolated InAs QDs fully confirm the predicted performances: the fraction of collected photons with the desired polarization can be as high as $95 \%$.

We first consider an infinite semiconductor photonic nanowire made of GaAs (refractive index $n_{\mathrm{sc}}=3.45$ ) and surrounded by an air cladding $\left(n_{c}=1\right)$. The wire features an elliptical section, whose major and minor diameters $d_{\|}$and $d_{\perp}$ are oriented along the $\|$ and $\perp$ directions, respectively. In the following, we assume a free-space operation wavelength $\lambda=950 \mathrm{~nm}$, and we suppose that $d_{\|}$and $d_{\perp}$ are small enough to ensure that the wire only supports two guided modes, $M_{\|}$and $M_{\perp}$. In the wire section, the dominating electric field components $E_{\|}\left(E_{\perp}\right)$ associated with $M_{\|}\left(M_{\perp}\right)$ is oriented along $\|(\perp)$. Adjusting the aspect ratio of the ellipse allows confining tightly $M_{\|}$inside the wire while $M_{\perp}$ is expelled in the air cladding. This effect, illustrated in Fig. 1 for $d_{\|}=\lambda / n_{\mathrm{sc}}$ and $d_{\perp}=d_{\|} / 2$, results from an interplay between the field continuity conditions, the anisotropic shape and the large refractive index contrast between the wire and the cladding. Across the wire sidewall, the tangential component of the electric field $\mathbf{E}$ and the normal component of the electric displacement $\mathbf{D}=\epsilon \mathbf{E}$ are continuous. Considering $M_{\perp}$, the second condition combined with the large index 

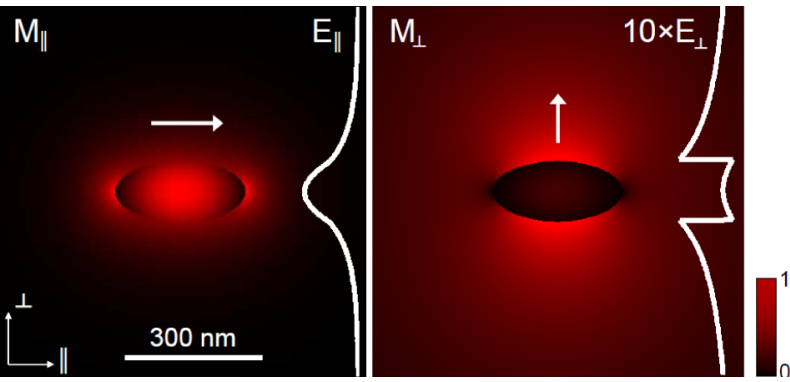

FIG. 1 (color online). Selective deconfinement of the guided mode $M_{\perp}$ in an elliptical wire $\left(d_{\|}=\lambda / n_{\mathrm{sc}}, d_{\perp}=d_{\|} / 2\right)$. The two color plots picture $E_{\|}$and $E_{\perp}$, the dominant in-plane electric field components associated with the modes $M_{\|}$and $M_{\perp}$, respectively. For clarity, $E_{\perp}$ has been multiplied by 10 . The linear plot on the right of the panels shows the evolution of $E_{\|}$and $10 \times E_{\perp}$ along the ellipse short axis. The arrows recall the mode polarization.

contrast imposes a strong discontinuity of $E_{\perp}$ along the short axis of the ellipse [Fig. 1]. The structure is then too thin to ensure a proper confinement of $M_{\perp}$, that is expelled in the cladding. In contrast, for $M_{\|}, E_{\|}$is continuous along the short axis and $d_{\perp}$ is compatible with a good mode confinement. Choosing $d_{\|}=\lambda / n_{\mathrm{sc}}$ further optimizes this confinement (note that $E_{\|}$is discontinuous along the long axis). Denoting $\Gamma_{M_{i}}^{j}(i, j=\|, \perp)$ the SE rate into the mode $M_{i}$ of a dipole source oriented along $j$, we have $\Gamma_{M_{\|}}^{\|} / \Gamma_{M_{\perp}}^{\perp}>100$ for on-axis dipoles. Moreover, $\Gamma_{M_{\|}}^{\|}$is very close to $\Gamma_{3 \mathrm{D}}$, the SE rate of the same dipole source immersed in bulk GaAs. Thanks to the good coupling to $M_{\|}$, the emitter brightness is preserved in the waveguide structure.

In addition, SE control in photonic nanowires also relies on a pronounced screening of the $3 \mathrm{D}$ continuum of socalled radiative modes [16]. To optimize this effect, we consider here an emitter that features dipole components perpendicular to the wire axis; this condition is easily achieved in a wide family of semiconductor heterostructures, including unstrained and compressively strained QWs and QDs. Polarization control at the SE level requires that each dipole component is simultaneously coupled to $M_{\|}$and $M_{\perp}$. Under this hypothesis, that will be further discussed at the end of the paper, the photons are emitted preferentially in $M_{\|}$, the fastest decay channel. For an emitter with two equal dipole components, the fraction $\beta_{\|}$of SE funneled into $M_{\|}$then reads $\beta_{\|}=\left(\Gamma_{M_{\|}}^{\|}+\right.$ $\left.\Gamma_{M_{\|}}^{\perp}\right) /\left(\Gamma_{\text {tot }}^{\|}+\Gamma_{\text {tot }}^{\perp}\right) ; \Gamma_{\text {tot }}^{j}$ is the total decay rate of a dipole source oriented along $j=\|, \perp$.

Considering the wire sketched in Fig. $1, \beta_{\|}$has been calculated numerically using a full-vectorial finite element method [17], for a embedded point emitter. When this emitter is located on the wire axis, Fig. 2(a) illustrates that $\beta_{\|} \approx 92 \%$ (for $\lambda=960 \mathrm{~nm}$ ). Moreover, $\beta_{\|}>90 \%$

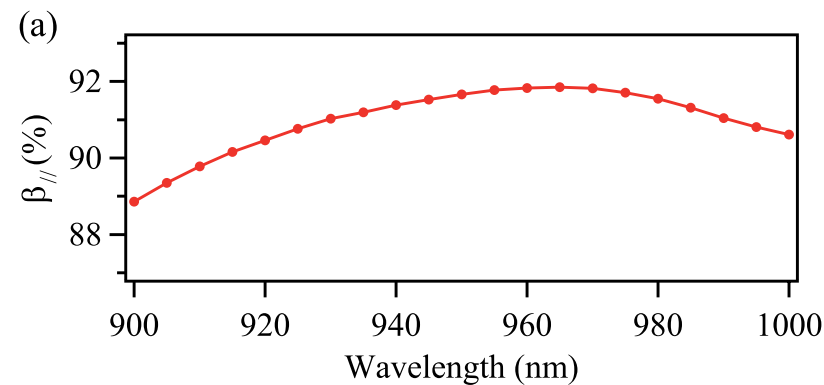

(b)

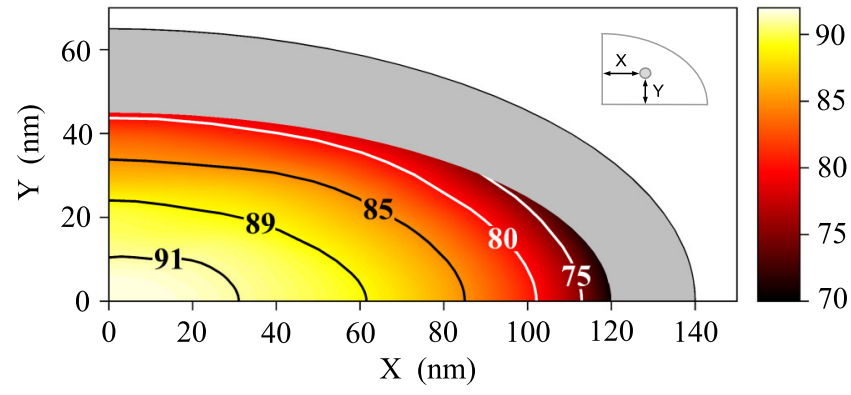

FIG. 2 (color online). Spontaneous emission control in an infinite photonic nanowire. The dimensions of the elliptical section are those of Fig. 1. (a) $\beta_{\|}$as a function of the free space operation wavelength $\lambda$ for an on-axis emitter. (b) $\beta_{\|}$for off-axis emitters $(\lambda=950 \mathrm{~nm})$. The calculation is conducted for an emitter-sidewall distance (solid black line) exceeding $20 \mathrm{~nm}$. For shorter distances (grey area), surface induced nonradiative processes will likely quench the radiative yield of the emitter.

over the 910-1000 nm wavelength range: such a broadband SE control is a key asset of the 1D photonic system under consideration. Figure 2(b) shows the calculated variation of $\beta_{\|}$versus the emitter position $(\lambda=950 \mathrm{~nm})$. In an infinite wire, $\sim 20 \%$ of a set of randomly positioned emitters display $\beta_{\|}>85 \%$. Such a tolerance, which is associated with the very efficient inhibition of SE into the radiative modes, is of major practical interest. Thus, elliptical photonic nanowires realize the ideal situation envisioned by Yablonovitch [18], without resorting to a complex photonic band gap structure.

The fabrication of elliptical photonic wires starts with a planar, nonintentionally doped structure which is grown by molecular beam epitaxy on a [001] GaAs wafer. It consists of a single layer of self-assembled InAs QDs buried $1 \mu \mathrm{m}$ below the top surface of a $2 \mu \mathrm{m}$-thick GaAs layer. A nickel hard mask is first defined using $e$-beam lithography, then the wires are etched with a carefully optimized dry etching step ( $\mathrm{SiCl}_{4}-\mathrm{Ar}$ plasma), and the nickel mask is finally removed by selective wet etching. The sample features a series of fields comprising ellipses with various dimensions and aspect ratios; the good control over the section geometry is illustrated in Fig. 3(b). In the laboratory frame, the crystal directions [110] and [1ํㅣㄹ are, respectively, horizontal $(h)$ and vertical $(v)$. The pattern was repeated for three different orientations $\theta_{\|}$of the ellipse major axis with respect to $(h): \theta_{\|}=0^{\circ}, 45^{\circ}$, and $90^{\circ}$. 
(a)
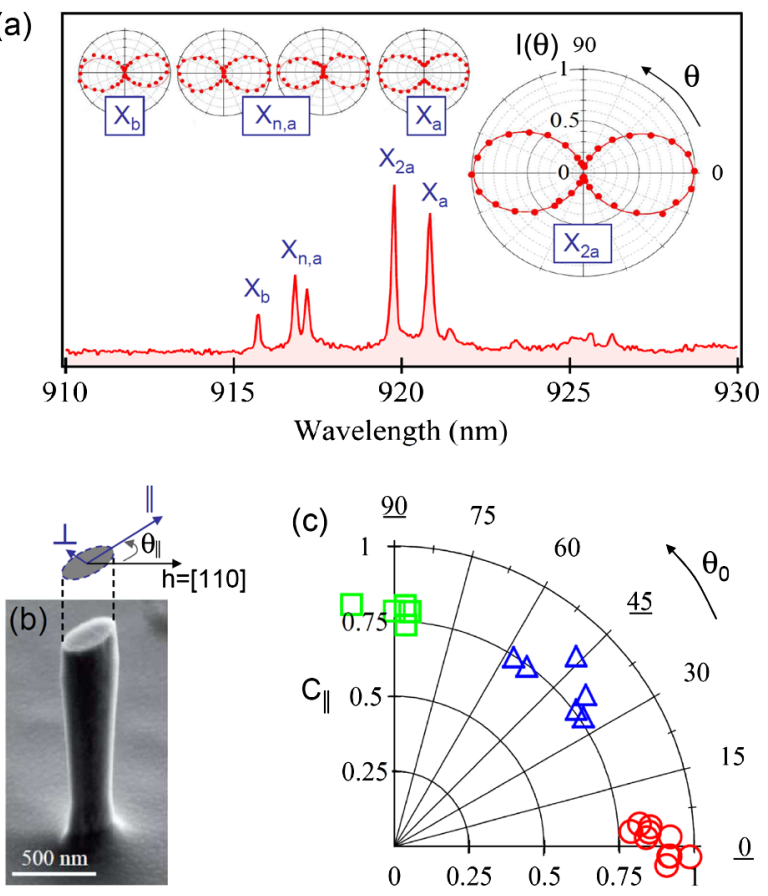

FIG. 3 (color online). Polarization control in elliptical photonic nanowires. (a) $\mu \mathrm{PL}$ spectra of $\mathrm{PW}_{0}\left[\theta_{\|}=0^{\circ}\right.$, i.e., $\|$ oriented along (h)]. The polarization analysis associated with the 5 isolated spectral lines is shown in the insets. The solid lines are fit using the expression given in the text. (b) Scanning electron microscope image of a typical fabricated structure. (c) Degree of linear polarization plotted against the polarization angle for emitters embedded in photonic wires with dimensions close to the one of $\mathrm{PW}_{0}$, but various ellipse orientations: $\theta_{\|}=0^{\circ}(\bigcirc), 45^{\circ}(\triangle)$, $90^{\circ}(\square)$.

The sample is mounted on the cold finger of a heliumflow cryostat with optical access. Nonresonant excitation is provided by a pulsed Ti:sapphire laser (wavelength: $870 \mathrm{~nm}, 200$ fs-pulses, repetition rate: $76 \mathrm{MHz}$, typical mean power: $5-100 \mu \mathrm{W}$ ), which is focused on a single nanowire with a microscope objective (N.A. $=0.6$ ). The laser generates electrons and holes in the 2D continuum of the QD wetting layer. The QD luminescence signal is then collected by the same objective, and analyzed with spectral $(\sim 150 \mathrm{pm})$ and polarization resolution. The residual polarization anisotropy of the setup was checked to be on the order of $1 \%$ above $900 \mathrm{~nm}$. In addition, the ensemble luminescence of the QDs studied in this work, which peaks around $930 \mathrm{~nm}$, does not exhibit any significant preferential polarization.

Figure 3(a) shows a micro-photoluminescence ( $\mu \mathrm{PL})$ spectrum recorded at $8 \mathrm{~K}$ from the elliptical wire $\mathrm{PW}_{0}$. The major axis of $\mathrm{PW}_{0}$ is oriented along the $(h)$ direction, with $d_{\|}=280 \mathrm{~nm}$ and $d_{\perp}=0.46 \times d_{\|}$. The spectrum features five spectrally isolated transitions, which have been identified using the dependence of the spectrally integrated intensity $I$ on the pumping power and decay time measurements. The lines are attributed to two different QDs named a and $\mathrm{b}$, and have been identified as neutral exciton $(X)$, biexciton $\left(X_{2}\right)$, and, likely, a higher energy excitonic complex $\left(X_{n}\right)$. The insets show the corresponding polarization analysis: $I$ is plotted against the polarization analysis angle $\theta$ [measured with respect to $(h)]$. Clearly, these five different lines exhibit a strong linear polarization, which is moreover closely aligned along the ellipse major axis. This is a first and strong evidence of an efficient polarization control by the photonic structure. For a quantitative analysis, the polarization data are fitted to the simple law: $I(\theta)=\frac{1}{2} \times$ $\left(I_{\|}+I_{\perp}\right)+\frac{1}{2}\left(I_{\|}-I_{\perp}\right) \cos \left(2 \theta-2 \theta_{0}\right)+B . I_{\|}$is the maximum intensity, obtained for $\theta=\theta_{0} ; I_{\perp}$ is the minimum intensity and $B$ accounts for the dark counts of the detector. As a figure of merit, we introduce $C_{\|}=I_{\|} /\left(I_{\|}+I_{\perp}\right)$, the fraction of collected photons linearly polarized along $\theta_{0}$. For the transition $X_{2 a}, C_{\|}$reaches 95\% [19]. Moreover, $C_{\|}$ exceeds $90 \%$ across the entire $5 \mathrm{~nm}$ wavelength range spanned by the 5 transitions: this is an experimental indication of the broadband SE control provided by elliptical photonic nanowires.

Highly polarized emission was collected from a number of structures with dimensions close to that of $\mathrm{PW}_{0}$, but various ellipse orientations: $\theta_{\|}=0^{\circ}, 45^{\circ}$, and $90^{\circ}$. The results are summarized in Fig. 3(c), which is a polar plot of $C_{\|}$versus $\theta_{0}$, for transitions studied in nine different wires. High $C_{\|}$are reproducibly obtained and the linear polarization of the light is imposed by the orientation of the photonic structure, regardless of the orientation of the GaAs crystal.

At this stage, it is instructive to examine the detailed mechanisms associated with polarization control for lowenergy excitonic complexes confined in a self-assembled InAs QD. Even in asymmetric QDs, charged excitons remain twofold degenerated, isotropic emitters: they fulfill the requirement of polarization control. The case of the neutral exciton is more subtle: the interplay between anisotropy and exchange interactions lifts the degeneracy of the two optically active transitions, with an energy splitting on the order of a few tens of $\mu \mathrm{eV}$ (not resolved in our setup). The associated eigenstates have a linear dipole oriented along the [110] and [110] crystal directions, and we assume they have similar oscillator strengths for simplicity. In that case, polarization control at the SE level can be achieved for a specific orientation of the photonic structure: if $\theta_{\|}=45^{\circ}$, each dipole is simultaneously coupled to $M_{\|}$and $M_{\perp}$. In contrast, if $\theta_{\|}=0^{\circ}$ or $90^{\circ}$, each dipole is coupled to one mode and decays independently, similarly to Ref. [20]. An additional coupling mechanism between the bright states is then required to achieve a preferential emission into $M_{\|}$. Interestingly enough, the neutral biexciton is unconditionally coupled to $M_{\|}$and $M_{\perp}$, also leading to the desired SE control [Fig. 4(b)].

A complementary evidence for single-mode emission is obtained from time-resolved $\mu$ PL. Figure 4(a) presents 


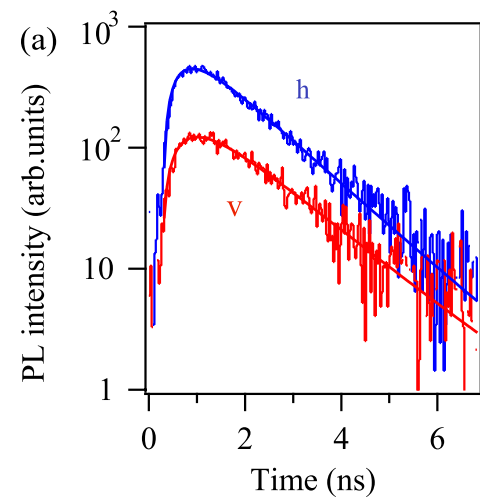

(b)

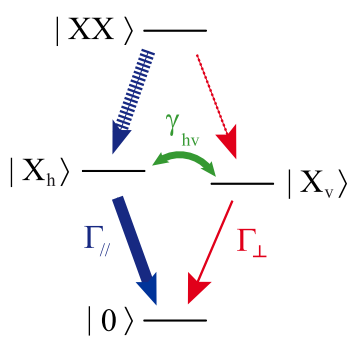

FIG. 4 (color online). Controlling the polarization of the neutral exciton. Measurements are taken on the line $X_{b}$ of sample $\mathrm{PW}_{0}$, whose major axis $\|$ is aligned on $(h)$. (a) Time-resolved decay, for collected polarization oriented along $\|$ and $\perp$ (log-lin scale). (b) Level structure and associated radiative rates. In this anisotropic photonic structure, $\Gamma_{\|} \gg \Gamma_{\perp}$. A fast spin-flip rate $\left(\gamma_{\mathrm{hv}} \gg \Gamma_{\|}\right)$can couple the bright excitons $\left|X_{h}\right\rangle$ and $\left|X_{v}\right\rangle$, leading to a single decay rate.

measurements carried out on the excitonic line $X_{b}$ of $\mathrm{PW}_{0}$ [ $\|$ and $\perp$ respectively aligned along $(h)$ and $(v)$, see Fig. 4(b)]. The decay for the analyzed polarizations \| and $\perp$ are monoexponential, with very similar decay times (1.22 and $1.39 \mathrm{~ns})$. This result, reproducibly obtained for all the excitonic lines, suggests a fast spin-flip mechanism between the bright components of the neutral exciton [21]. They are then effectively coupled to $M_{\|}$and $M_{\perp}$, decay with a single radiative rate and the light emitted by the structure is linearly polarized along $\|$.

In quantum communications, the present work paves the way for the realization of a bright source of single photons with well defined polarization, provided an appropriate engineering of the wire ends is conducted [15]. Furthermore, this approach could be applied for the efficient generation of indistinguishable photons. Given the moderate SE rate enhancement provided in a photonic nanowire with an integrated bottom mirror $(\times 1.7)$, this will likely require the integration of a fast quantum emitter, such as a large QD formed by the thickness fluctuations of the interfaces of a quantum well [22]. Finally, recent theoretical investigations have also shown that a 3-level emitter strongly coupled to a 1D continuum of optical modes can mediate a strong photon-photon interaction, opening appealing perspective for the development of waveguide-based photonic quantum gates [23-25].

Elliptical photonic nanowires also offer the opportunity to improve the performance of standard nanowire lasers [26]. A nanocavity could be defined by the integration of a bottom and a top mirror [27] and a high $\beta$ can be obtained for both QD and QW gain medium. Finally, a trumpetlike tapering of the upper end of the wire provides a directive far-field emission [28] and introduces a large top facet that is also convenient to implement a high reflectivity mirror.
To conclude, we have demonstrated that a dielectric photonic nanowire with an elliptical section defines an electromagnetic environment dominated by a single guided mode with a linear polarization. We have also discussed the large application spectrum of this truly monomode 1D photonic system.

The authors thank M. Ducruet for the epitaxial growth of the sample. Financial support from the "Nanosciences aux limites de la Nanoélectronique" Fondation is acknowledged. Clean room processing was performed in the "Plateforme Technologique Amont" and CEA LETI MINATEC/DOPT clean rooms.

*julien.claudon@cea.fr

[1] E. M. Purcell, Phys. Rev. 69, 674 (1946).

[2] F. Albert, T. Braun, T. Heindel, C. Schneider, S. Reitzenstein, S. Höfling, L. Worschech, and A. Forchel, Appl. Phys. Lett. 97, 101108 (2010).

[3] B. Ellis, M. A. Mayer, G. Shambat, T. Sarmiento, J. Harris, E. E. Haller, and J. Vuckovic, Nature Photon. 5, 297 (2011).

[4] E. Moreau, I. Robert, J. M. Gérard, I. Abram, L. Manin, and V. Thierry-Mieg, Appl. Phys. Lett. 79, 2865 (2001).

[5] W. H. Chang, W. Y. Chen, H. S. Chang, T. P. Hsieh, J. I. Chyi, and T. M. Hsu, Phys. Rev. Lett. 96, 117401 (2006).

[6] S. Strauf, N. G. Stoltz, M. T. Rakher, L. A. Coldren, P. M. Petroff, and D. Bouwmeester, Nature Photon. 1, 704 (2007).

[7] Q. Turchette, R. Thompson, and H. Kimble, Appl. Phys. B 60, S1 (1995).

[8] H. J. Kimble, Nature (London) 453, 1023 (2008).

[9] D. Englund, D. Fattal, E. Waks, G. Solomon, B. Y. Zhang, T. Nakaoka, Y. Arakawa, Y. Yamamoto, and J. Vuckovic, Phys. Rev. Lett. 95, 013904 (2005).

[10] J. P. Zhang, D. Y. Chu, S. L. Wu, S. T. Ho, W. G. Bi, C. W. Tu, and R. C. Tiberio, Phys. Rev. Lett. 75, 2678 (1995).

[11] J. Claudon, J. Bleuse, N. S. Malik, M. Bazin, P. Jaffrennou, N. Gregersen, C. Sauvan, P. Lalanne, and J.-M. Gérard, Nature Photon. 4, 174 (2010).

[12] E. Viasnoff-Schwoob, C. Weisbuch, H. Benisty, S. Olivier, S. Varoutsis, I. Robert-Philip, R. Houdré, and C. J.M. Smith, Phys. Rev. Lett. 95, 183901 (2005).

[13] T. Lund-Hansen, S. Stobbe, B. Julsgaard, H. Thyrrestrup, T. Sünner, M. Kamp, A. Forchel, and P. Lodahl, Phys. Rev. Lett. 101, 113903 (2008).

[14] A. Akimov, A. Mukherjee, C. L. Yu, D. E. Chang, A. S. Zibrov, P. R. Hemmer, H. Park, and M. D. Lukin, Nature (London) 450, 402 (2007).

[15] I. Friedler, C. Sauvan, J. P. Hugonin, P. Lalanne, J. Claudon, and J. M. Gérard, Opt. Express 17, 2095 (2009).

[16] J. Bleuse, J. Claudon, M. Creasey, N.S. Malik, J.-M. Gérard, I. Maksymov, J.-P. Hugonin, and P. Lalanne, Phys. Rev. Lett. 106, 103601 (2011).

[17] Y. Chen, T. R. Nielsen, N. Gregersen, P. Lodahl, and J. Mørk, Phys. Rev. B 81, 125431 (2010).

[18] E. Yablonovitch, Phys. Rev. Lett. 58, 2059 (1987).

[19] The diffraction of the well-confined mode $M_{\|}$is much stronger than that of $M_{\perp}$, which is deconfined in the air 
cladding. Thus, even higher $C_{\|}$should be obtained with a proper tapering of the wire upper end.

[20] Q. Wang, S. Stobbe, H. Thyrrestrup, H. Hofmann, M. Kamp, T. W. Schlereth, S. Höfling, and P. Lodahl, Opt. Lett. 35, 2768 (2010).

[21] I. Favero, G. Cassabois, C. Voisin, C. Delalande, P. Roussignol, R. Ferreira, C. Couteau, J.P. Poizat, and J. M. Gérard, Phys. Rev. B 71, 233304 (2005).

[22] J. Hours, S. Varoutsis, M. Gallart, J. Bloch, I. RobertPhilip, A. Cavanna, I. Abram, F. Laruelle, and J. M. Gérard, Appl. Phys. Lett. 82, 2206 (2003).
[23] J.-T. Shen and S. Fan, Phys. Rev. Lett. 98, 153003 (2007).

[24] D. Roy, Phys. Rev. Lett. 106, 053601 (2011).

[25] P. Kolchin, R. F. Oulton, and X. Zhang, Phys. Rev. Lett. 106, 113601 (2011).

[26] J. C. Johnson, H. Yan, P. Yang, and R. J. Saykally, J. Phys. Chem. B 107, 8816 (2003).

[27] I. Friedler, P. Lalanne, J. P. Hugonin, J. Claudon, J. M. Gérard, A. Beveratos, and I. Robert-Philip, Opt. Lett. 33, 2635 (2008).

[28] N. Gregersen, T. R. Nielsen, J. Mørk, J. Claudon, and J.-M. Gérard, Opt. Express 18, 21204 (2010). 